\title{
DOIS MODELOS CONTEMPORÂNEOS DE DIVULGAÇÃO FINANCEIRA NA INDÚSTRIA BANCÁRIA BRASILEIRA: QUAL É MAIS CONSERVADOR?
}

\author{
Everton Souza da Cunha ${ }^{1}$ \\ José Alves Dantas ${ }^{2}$ \\ Otávio Ribeiro Medeiros ${ }^{3}$
}

\begin{abstract}
Resumo: O presente estudo teve por objetivo identificar qual dos modelos contábeis adotados, contemporaneamente, pelas instituições financeiras brasileiras - Cosif ou IFRS - apresenta de forma mais relevante a característica do conservadorismo contábil condicional. Considerando que o Cosif, instituído pelos reguladores, deve atender prioritariamente aos interesses de supervisão prudencial do sistema e das entidades, e que o modelo IFRS prioriza, declaradamente, o propósito de atender às necessidades informacionais de investidores e credores, é natural se esperar que esses modelos produzam características informacionais diferenciadas, entre as quais o conservadorismo. Para esse fim, foi utilizado o modelo de componentes transitórios, desenvolvido por Basu (1997) e ampliado por Ball e Shivakumar (2005), tendo como amostra dados das demonstrações financeiras anuais, de 2010 a 2014, de 41 instituições. Os resultados dos testes empíricos demonstraram que, nas demonstrações elaboradas com base no padrão Cosif, as variações positivas no resultado contábil não eram persistentes, contrariando as premissas do conservadorismo condicional. Por outro lado, foram constatadas as características do conservadorismo contábil condicional nas demonstrações no padrão IFRS. A combinação dessas evidências conduziu à não rejeição da hipótese de pesquisa que previa que as demonstrações financeiras anuais das instituições bancárias brasileiras apresentadas em padrão IFRS apresentam maior conservadorismo que as elaboradas no padrão Cosif.
\end{abstract}

Palavras-chave: Conservadorismo condicional. IFRS. Cosif. Bancos. Modelo de Basu. Brasil.

\footnotetext{
E-mail: dacunha.everton@gmail.com - Fucape Business School.

2 E-mail: josealvesdantas@unb.br - Universidade de Brasília

${ }^{3}$ E-mail: otavio@unb.br - Universidade de Brasília

- DOI: http://dx.doi.org/10.14392/asaa.2016090304

- Artigo submetido em: 20/09/2015. Aceito em: 22/11/2016
} 


\title{
TWO CONTEMPORANEOUS MODELS OF FINANCIAL DISCLOSURE IN THE BRAZILIAN BANKING INDUSTRY: WHICH IS MORE CONSERVATIVE?
}

\begin{abstract}
This study is aimed at identifying which of the accounting models currently adopted by Brazilian financial institutions - Cosif or IFRS - presents more clearly the characteristics of conditional accounting conservatism. Considering that Cosif, which is set up by regulators, should give priority to the interests of prudential supervision of the system and its entities, and that the IFRS model gives priority to attending the informational needs of investors and creditors, it is natural to expect these models to produce distinct informational characteristics from each other, among them, conservatism. For this purpose, the model of transient components, developed by Basu (1997) and augmented by Ball and Shivakumar (2005), was used, taking as sample data from the annual financial statements of 41 institutions, from 2010 to 2014. The results of the empirical tests showed that, in the statements based on the Cosif standards, the positive changes in accounting earnings were not persistent, contrary to the assumptions related to conditional conservatism. On the other hand, the characteristics of the conditional accounting conservatism in the IFRS financial statements were verified. The combination of these findings led to the non-rejection of the research hypothesis predicting that the annual financial statements of Brazilian banking institutions based on the IFRS standards present more conservatism than those based on the Cosif standards.
\end{abstract}

Keywords: Conditional conservatism. IFRS. Cosif. Banks. Basu's model. Brazil. 


\section{INTRODUÇÃO}

$\mathrm{N}$ o Brasil, por força Resolução n 3.786, de 24 de setembro de 2009, do Conselho Monetário Nacional [CMN], as instituições financeiras constituídas sob a forma de companhia aberta ou que sejam obrigadas a constituir comitê de auditoria devem divulgar dois conjuntos de demonstrações financeiras, elaboradas com base em modelos contábeis distintos: o primeiro, fundamentado no Plano Contábil das Instituições do Sistema Financeiro Nacional [Cosif], sob responsabilidade do Banco Central do Brasil [BCB]; o segundo, com base nas International Financial Reporting Stardars [IFRS], editadas pelo International Accounting Standards Board [IASB].

O presente estudo teve como objetivo identificar qual desses modelos contábeis, Cosif ou IFRS, apresenta de forma mais relevante a característica do conservadorismo contábil condicional na indústria bancária brasileira. A premissa é que esses modelos têm prioridades distintas - o Cosif, instituído pelos reguladores, deve atender prioritariamente aos interesses de supervisão sobre a estabilidade do sistema e a solidez das entidades, enquanto o modelo IFRS prioriza, declaradamente, as necessidades informacionais de investidores e credores - e, portanto, devem produzir informações financeiras com características diferenciadas. Entre essas características pode se destacar a do conservadorismo condicional, definido por Ball, Kothari e Robin (2000) como conservadorismo ex-post ou conservadorismo de resultado, que implica uma assimetria no reconhecimento de boas e más notícias nos resultados.

Apesar de outros estudos na literatura já terem abordado o tema conservadorismo contábil na indústria bancária brasileira - como Sterzeck (2011), Brito, Lopes e Coelho (2012) e Dantas, Paulo e Medeiros (2013), por exemplo - nenhum deles tratou especificamente dessa dicotomia decorrente da dupla evidenciação, com base em modelos contábeis distintos. Mesmo trabalhos que avaliam o impacto dos modelos contábeis no conservadorismo das informações financeiras, geralmente o fazem em perspectiva, ou seja, avaliam o impacto da mudança dos modelos. É o caso, por exemplo, dos estudos que avaliaram os efeitos da adoção dos padrões IFRS no conservadorismo condicional, como Santos, Lima, Freitas \& Lima (2011), Rodrigues (2012) e Filipin, Teixeira, Bezerra \& Cunha (2012).

Nesse sentido, a abordagem deste estudo pode ser considerada como inovadora, por avaliar os efeitos dos dois modelos, contemporaneamente. Como essa condição da dupla divulgação financeira é não usual, o sistema bancário brasileiro oferece uma oportunidade privilegiada de se empreender pesquisas do gênero, mitigando-se o risco de as mudanças nos eventos econômicos justificarem alterações nas características das informações financeiras que não sejam necessariamente identificadas nos modelos que avaliam as informações contábeis em perspectivas - mudança de modelo contábil a partir de determinada data-base.

Outras contribuições de estudos dessa natureza podem ser associadas aos chamados efeitos colaterais da dupla evidenciação na indústria bancária brasileira, tanto para os usuários quanto para o próprio regulador, sobre qual seria a melhor expressão da real situação econômico-financeira da entidade que divulga. Por outro lado, também é possível se refletir sobre a eventual complementariedade dessas 
informações, considerando que um modelo pode produzir informações mais objetivas e outro informações mais úteis, representando certo balanceamento entre esses dois aspectos, conforme destacado por Dantas, Rodrigues, Niyama \& Mendes (2010) e Paulo, Carvalho \& Girão (2014) em estudo sobre a dicotomia entre normatização baseada em princípios, regras e objetivos.

Isso aumenta a relevância de se entender os efeitos dos modelos na produção das informações contábeis. Dependendo do acúmulo de evidências empíricas, é possível, inclusive, que no futuro o próprio regulador venha a avaliar a conveniência de se adotar o padrão IFRS, inclusive para fins prudenciais, considerando, inclusive, a relação custo-benefício dessa dupla divulgação. Este estudo contribui com o desenvolvimento dessa literatura, entendendo que os resultados ainda são embrionários, até porque o horizonte temporal é relativamente curto - início em 2010.

Quanto à opção por se concentrar no exame do conservadorismo, isso se justifica por ser uma característica particularmente relevante no sistema bancário, em que a confiança e a credibilidade são condições primordiais de funcionamento. De se ressaltar que embora a literatura apresente críticas ao conservadorismo contábil, principalmente no que se refere à sua eventual incompatibilidade com a característica da neutralidade da informação contábil, a sua utilidade se sustenta em argumentos como: fornecer informações mais confiáveis aos investidores, tendo em vista que as informações extremamente otimistas seriam evitadas (Lopes, 2002); quando se tenta atingir a "neutralidade da informação", em detrimento do conservadorismo, sem entender as razões pelas quais o ato prudencial passou a existir, a tendência é falhar e produzir consequências inesperadas (Watts, 2003a, 2003b); reduz a assimetria da informação, pois restringe a habilidade de a administração manipular as informações financeiras e traz um ambiente que amplia as fontes de informação (Amaral, Riccio \& Sakata (2012).

A realização dos testes empíricos teve por base a aplicação do modelo de Basu (1997) ampliado por Ball e Shivakumar (2005), utilizando as informações das demonstrações financeiras anuais apresentadas pelas instituições bancárias que apresentaram suas demonstrações nos dois padrões, Cosif e IFRS, de 2010, quando começou a ser exigida a divulgação das demonstrações em IFRS, a 2014. Os resultados dos testes demonstraram que, nas demonstrações elaboradas com base no padrão Cosif, as variações positivas no resultado contábil não eram persistentes, contrariando as premissas do conservadorismo condicional. Por outro lado, foram constatadas as características do conservadorismo contábil condicional nas demonstrações no padrão IFRS. A combinação dessas evidências conduziu à não rejeição da hipótese de pesquisa que previa que as demonstrações financeiras anuais das instituições bancárias brasileiras apresentadas em padrão IFRS apresentam maior conservadorismo que as elaboradas no padrão Cosif.

Além dessa parte introdutória, que contextualiza o tema e define o propósito e o alcance da pesquisa, o presente estudo contempla: a revisão de literatura sobre o tema, incluindo pesquisas relacionadas ao conservadorismo contábil nas instituições financeiras bancárias (Seção 2); a descrição dos procedimentos metodológicos para a realização dos testes empíricos, incluindo o desenvolvimento e formulação das hipóteses de pesquisa (Seção 3); a análise e a discussão dos resultados (Seção 4); e as considerações finais, considerando a comparação entre as referências teóricas e os achados empíricos (Seção 5).

\section{REFERENCIAL TEÓRICO}

\subsection{CONSERVADORISMO CONTÁBIL}

Para Most (1982), o conservadorismo é definido como uma tendência de subavaliar, de forma deliberada, mas consistente, o patrimônio líquido e os lucros de uma entidade. Tem como característica 
a prudência, associada a um enfoque menos otimista, quando da escolha entre duas alternativas de valores com a mesma probabilidade a serem pagos ou recebidos no futuro. Ball, Kothari e Robin (2000) destacam que o conservadorismo contábil pode ser definido de dois modos: (i) o conservadorismo incondicional (ex-ante) ou conservadorismo patrimonial, no qual se deve utilizar o menor valor atual para ativos e receitas e maiores valores para passivos e despesas, mantendo a subavaliação sistemática do patrimônio; e (ii) o conservadorismo condicional (ex-post) ou conservadorismo de resultado, que implica uma assimetria no reconhecimento dos efeitos causados por boas e más notícias nos resultados.

Para Rodrigues (2012), a tempestividade do reconhecimento das perdas econômicas é uma característica determinante para a qualidade dos resultados. O reconhecimento das más notícias é mais oportuno do que o reconhecimento das boas notícias. Essa premissa é fortemente defendida quando se fala em conservadorismo, pois os ganhos econômicos, por terem seu reconhecimento mais persistente ao longo do tempo são menos impactantes no resultado imediato, Em contrapartida, as perdas decorrentes das notícias desfavoráveis provocam impactos imediatos, significando que essas perdas são incorporadas ao resultado presente e configurando a transitoriedade dos efeitos das notícias negativas nos resultados (Dantas, Paulo \& Medeiros, 2013).

1 Tratando do conservadorismo nos resultados contábeis, Coelho e Lima (2008) avaliaram se há demanda diferenciada por dados contábeis entre usuários de informações ligados a companhias de capital aberto e a empresas de capital fechado, comparando-as na métrica do conservadorismo contábil. Os resultados apresentados indicaram a carência de atributos de qualidade - conservadorismo - requeridos para atender aos usuários que portam contratos de fornecimento de recursos ou de governança com as sociedades por ações brasileiras. Em consonância com o resultado, há de se destacar que os acionistas das companhias abertas têm proteção mais eficaz em função da atuação da autarquia reguladora do mercado de capitais, com interpretações legais favoráveis aos minoritários (Black, 2001).

No estudo de Brito e Martins (2013), a finalidade foi investigar se a adoção de práticas contábeis conservadoras leva à redução no custo do crédito bancário das empresas no Brasil. Os testes empíricos tiveram por base uma amostra de 1.300 empresas e de 813 mil contratos de crédito, no período de 2000 a 2009. Os autores não obtiveram evidências de relação estatística significativa entre a métrica do conservadorismo e as taxas de juros das operações de crédito, o que levou à não rejeição da hipótese formulada de que as empresas que utilizam práticas conservadoras não obtêm crédito bancário a custos mais baixos.

\subsection{CONSERVADORISMO CONTÁBIL NA INDÚSTRIA BANCÁRIA}

Se o conservadorismo pode ser um aspecto importante a se considerar para entender o uso e o impacto das informações contábeis, bem como a matriz de incentivos da administração na preparação das demonstrações financeiras, no caso das entidades bancárias isso pode ser ainda mais relevante. Isso porque são entidades que dependem fundamentalmente da confiança e credibilidade para poderem exercer em sua plenitude a função de intermediadores financeiros, o que pode ser um incentivo para demonstrarem atuar com conservadorismo na divulgação. Por outro lado, os bancos estão sujeitos a forte regulação estatal, incluindo requerimentos de capital mínimo, o que pode representar um incentivo ao comportamento inverso ao conservadorismo condicional.

Não obstante esse contexto, apesar do tema conservadorismo condicional ser relativamente comum na literatura contábil, estudos direcionados à avaliação da prática prudencial na indústria bancária po- 
dem ser, segundo Dantas, Paulo e Medeiros (2013), considerados incipientes, principalmente em termos nacionais. Em âmbito internacional podem ser citados como exemplos Nichols, Wahlen \& Wieland (2009), Lima, Fonseca e Brito (2009) e Tapia, Sánchez, Alemán \& Fernandez (2011), Kanagaretnam, Lim \& Lobo (2014) e Lim, Lee, Kausar \& Walker (2014). No Brasil, foram identificados os estudos de Sterzeck (2011), Brito, Lopes e Coelho (2012) e Dantas, Paulo e Medeiros (2013).

Nichols, Wahlen \& Wieland (2009) identificaram maior grau de conservadorismo contábil dos bancos com capital aberto que dos bancos com capital fechado, ou seja, os bancos listados em bolsa reconhecem mais tempestivamente as perdas e menos oportunamente os ganhos, quando comparados com os bancos de capital fechado. Essa mesma hipótese foi testada por Lima, Fonseca e Brito (2009), no âmbito do sistema bancário português, mas os resultados não confirmaram essa perspectiva.

A influência do nível de capital das instituições bancárias em relação à prática do conservadorismo condicional foi examinada por Tapia et al. (2011), encontrando uma relação negativa entre essas variáveis. $\mathrm{Na}$ ótica dos pesquisadores, a potencial explicação para esses resultados seria que bancos com menor nível de capital estão sujeitos a uma maior vigilância, o que justificaria a adoção de um maior grau de conservadorismo condicional, para minimizar os custos de litígios e a probabilidade de ações políticas regulatórias adversas.

A influência da cultura nacional no conservadorismo contábil praticado pela indústria bancária foi um dos aspectos examinados por Kanagaretnam, Lim \& Lobo (2014). Usando o individualismo e a aversão à incerteza como proxies da cultura nacional, os autores constataram que o nível de conservadorismo praticado pelos bancos é negativamente relacionado com o individualismo e positivamente associado à aversão à incerteza.

Lim et al. (2014), por sua vez, investigaram os efeitos do conservadorismo contábil no reconhecimento de perdas nas operações de crédito por parte dos bancos, concluindo que aqueles que são mais tempestivos no reconhecimento de perdas cobram spreads maiores. Esses achados são consistentes, no entendimento dos autores, com o argumento de que o conservadorismo condicional funciona como um instrumento de governança. Em síntese, bancos que são mais tempestivos no reconhecimento de perdas atuam de forma mais prudente e têm comportamento menos pró-cíclico em momentos de crise.

No Brasil, foram identificados os estudos de Sterzeck (2011), Brito, Lopes e Coelho (2012) e Dantas, Paulo e Medeiros (2013).

Sterzeck (2011) abordou o efeito da convergência contábil investigando se houve alteração no conservadorismo condicional das instituições financeiras de capital aberto que estavam listadas na BM\&FBovespa após a vigência da Lei 11.638/2007 e da Resolução CMN n 3.786/2009. O pressuposto considerado é que os órgãos reguladores estimulam a utilização do conservadorismo nas informações contábeis, havendo incentivos por parte dos reguladores para que as empresas apresentem resultados mais conservadores. Essa forma de apresentação das demonstrações contábeis visa mitigar o risco sistêmico, definido por Capelletto (2006) como o grau de incerteza existente no sistema, resultante de alterações nos níveis de risco de crédito, de taxa de juros e da taxa de câmbio. Os testes empíricos produziram resultados inconclusivos, não sendo possível inferir que os lucros contábeis foram afetados pela adoção da nova Lei, ou após a vigência da Resolução.

Tendo por objetivo investigar a presença de conservadorismo condicional nos lucros evidenciados pelas instituições financeiras no Brasil, Brito et al. (2012), examinaram se a prática prudencial apresenta diferenças entre bancos estatais e bancos privados. Utilizando dados relativos a 260 bancos abrangendo o período de 1997 a 2010, os autores encontraram, inicialmente, indícios de que os lucros contábeis reproduzidos pelos bancos brasileiros apresentam características de conservadorismo condicional. 
Porém, a pesquisa apresentou uma análise secundária na qual os autores identificaram que os bancos estatais apresentaram maiores níveis de conservadorismo com relação aos bancos privados. Uma justificativa plausível, segundo os pesquisadores, seria o fato de os gestores dos bancos estatais terem de tomar decisões sobre a alocação de recursos públicos. Essa hipótese foi considerada como resultado da regulação e fiscalização por diversos órgãos de controle do governo.

Para Dantas et al. (2013), as variações positivas no resultado contábil são persistentes, enquanto as variações negativas são mais transitórias. Eles investigaram o conservadorismo condicional na indústria bancária brasileira em situações de maior percepção de risco, obtendo evidências de que as perdas econômicas relacionadas ao menor nível de participação do capital próprio são reconhecidas mais tempestivamente. Constataram, também, que não existem diferenças significativas no comportamento conservador praticado pelos bancos públicos e privados no Brasil.

\subsection{CONSERVADORISMO CONDICIONAL EM RELAÇÃO AOS MODELOS CONTÁBEIS}

Mesmo com a contribuição à literatura sobre o conservadorismo condicional na indústria bancária destacada na Seção 2.2, ainda há um campo vasto para explorar, particularmente no mercado brasileiro.

Nas pesquisas realizadas no Brasil, o conservadorismo condicional bancário foi analisado investigando-se: (i) se houve alteração no conservadorismo condicional após a vigência da Lei 11.638/2007 e da Resolução CMN no 3.786/2009, por Sterzeck (2011), para um período compreendido após a adoção da referida Lei; (ii) se a prática prudencial apresenta diferenças entre os bancos estatais e os bancos privados, por Brito et al. (2012), segmentando o período em anterior e posterior à adoção das normas internacionais; e (iii) se a presença de conservadorismo condicional na indústria bancária brasileira em situações de maior percepção de risco, também segmentado em períodos antes e depois da vigência das normas emitidas pelo IASB. Como se observa, mesmo os estudos que procuram avaliar os efeitos de modelos contábeis no nível de conservadorismo dos bancos brasileiros o fazem sempre em uma perspectiva temporal, ou seja, se houve mudança de comportamento a partir de determinada diretriz legal ou normativa.

O presente estudo, ao contrário, teve por foco a avaliação comparativa um período comum para as diferentes formas de demonstrações para as mesmas instituições, constituindo-se, portanto, em uma abordagem metodológica inovadora, só possível no contexto brasileiro, que impõe a um número de bancos o duplo disclosure financeiro. $\mathrm{O}$ objetivo da pesquisa concentra-se em identificar qual dos modelos contábeis adotados pelas instituições bancárias brasileiras para divulgar suas demonstrações financeiras - Cosif ou IFRS - representa de forma mais relevante a característica do conservadorismo contábil condicional.

Considerando a característica dos incentivos para a prática do conservadorismo na indústria bancária e o fato de que os modelos contábeis adotados no Brasil para a divulgação financeira dessas instituições apresentam características bem distintas - o Cosif pode ser definido como essencialmente baseado em regras e as IFRS em princípios - é natural se esperar que apresentem resultados diferenciados em relação ao conservadorismo condicional.

Uma primeira perspectiva pode ser construída a partir dos fundamentos teóricos apresentados e discutidos por Santos et al. (2011), Rodrigues (2012) e Filipin et al. (2012), que estudaram o conservadorismo relacionado aos padrões internacionais de contabilidade, e os resultados obtidos, principalmente por Filipin et al. (2012), que induzem à vinculação das normas internacionais, visando proteção ao usuário da 
informação através do conservadorismo. Nesse contexto, é formulada a seguinte hipótese de pesquisa, a ser testada empiricamente:

H1: As demonstrações financeiras anuais das instituições bancárias brasileiras apresentadas em IFRS são mais conservadoras que as demonstrações elaboradas no padrão Cosif.

Alternativamente, de acordo com os pressupostos teóricos abordados e comentados por Sterzeck (2011), Brito et al. (2012) e Dantas et al. (2013), que pesquisaram o conservadorismo sobre a ótica das instituições financeiras, com destaque para Sterzeck (2011) e Brito et al. (2012), que apontam a regulação bancária como um incentivo para se adotar o conservadorismo nas demonstrações financeiras, é possível se esperar que o modelo contábil definido pelo regulador apresente resultados mais conservadores do que o modelo IFRS. Isso dá sustentação à seguinte hipótese de pesquisa, a ser testada:

H2: As demonstrações financeiras anuais das instituições bancárias brasileiras apresentadas em padrão Cosif são mais conservadoras que as demonstrações elaboradas em IFRS.

Como se percebe, as hipóteses são alternativas e excludentes entre si, caracterizando a dubiedade implícita em relação aos argumentos teóricos e incentivos para o conservadorismo condicional por parte da indústria bancária, nos dois modelos contábeis.

\section{PROCEDIMENTOS METODOLÓGICOS}

\subsection{O MODELO DE COMPONENTES TRANSITÓRIOS DE BASU}

Para testar as hipóteses de pesquisa, seguindo Santos et al. (2011), Rodrigues (2012), Filipin et al. (2012), Sterzeck (2011), Brito et al. (2012) e Dantas et al. (2013), é utilizado o modelo de componentes transitórios do lucro, desenvolvido por Basu (1997) e ampliado por Ball e Shivakumar (2005). O modelo, em sua versão básica, foi desenvolvido para avaliar o conservadorismo condicional por meio da transitoriedade do lucro e tem a seguinte especificação:

$$
\Delta L L_{i t}=\alpha_{0}+\alpha_{1} D_{i t-1}+\alpha_{2} \Delta L L_{i t-1}+\alpha_{3}\left(D_{i t-1} * \Delta L L_{i t-1}\right)+\varepsilon_{i t}
$$

em que: $\Delta \mathrm{LL}_{\mathrm{it}}$ corresponde à variação do lucro líquido da i-ésima instituição no período $\mathrm{t}$, apurada anualmente, ponderada pelos ativos totais do início do período; $a_{j}, j=1, \ldots, 3$, são os parâmetros da regressão, $D_{i t}$ é variável dummy referente à i-ésima instituição no período t, que assume valor 1 quando $\Delta L L$ é negativa e 0 para os demais períodos; e $\varepsilon_{i t}$ é o termo de erro correspondente à i-ésima instituição no período t, com a premissa de distribuição normal e variância constante.

De acordo com as especificações de Basu (1997), para que seja confirmada a existência de conservadorismo condicional, é necessário observar os resultados obtidos empiricamente para o valor e o sinal do coeficiente $a_{2^{\prime}}$ que é o coeficiente relativo aos ganhos econômicos, e do coeficiente $a_{3^{\prime}}$ relativo às perdas econômicas.

Sob a perspectiva do conservadorismo condicional, espera-se que as perdas econômicas sejam reconhecidas de forma tempestiva, provocando transitoriedade nas variações negativas no resultado, ou seja, que o impacto das perdas apuradas seja revertido no próximo período. Consequentemente, espera-se que o sinal do coeficiente $a_{3}$ seja negativo.

Para o coeficiente $a_{2}$ há dois tipos de resultado que atendem à condição do conservadorismo condicional: i) de acordo com Nichols, Wahlen e Wieland (2009) e Tapia, Sánchez, Alemán e Fernández (2011), 
espera-se que o reconhecimento dos ganhos econômicos seja diferido, ou seja, que as variações positivas no resultado contábil sejam constantes, ocasionando sinal positivo; ii) segundo Ball e Shivakumar (2005), esse diferimento dos ganhos deve implicar que o coeficiente não seja estatisticamente diferente de zero, com a soma dos coeficientes $a_{2}$ e $a_{3}$ sendo menor que zero $\left(a_{2}+a_{3}<0\right)$.

Assim, a confirmação da condição do conservadorismo condicional nos resultados contábeis está relacionada à constatação de: valores negativos para o coeficiente $a_{3}$; valores estatisticamente nulos ou positivos para $a_{2}$; e que a soma dos coeficientes $a_{2}$ e $a_{3}$ sendo menor que zero. No caso de os dois modelos contábeis examinados atenderem a essas condições, a verificação de qual deles apresenta maior grau de conservadorismo condicional dependerá dos valores obtidos para os dois coeficientes.

Para o caso específico da condição de que a soma dos coeficientes $a_{2}$ e $a_{3}$ seja menor que zero, será utilizado como referência o procedimento baseado em Uriel (2013), que consiste em testar a hipótese nula de que $a_{2}+a_{3}=0$ contra a hipótese alternativa de $a_{2}+a_{3}<0$. Para isso, é desenvolvido o modelo de teste, partindo-se da seguinte condição:

$$
\begin{gathered}
\alpha_{2}+\alpha_{3}=\phi \rightarrow \alpha_{3}=\phi-\alpha_{2} \\
\text { Substituindo essa condição no modelo (3.1), tem-se: } \\
\Delta L L_{i t}=\alpha_{0}+\alpha_{1} D_{i t-1}+\alpha_{2} \Delta L L_{i t-1}+\left(\phi-\alpha_{2}\right)\left(D_{i t-1} * \Delta L L_{i t-1}\right)+\varepsilon_{i t} \\
\Delta L L_{i t}=\alpha_{0}+\alpha_{1} D_{i t-1}+\alpha_{2} \Delta L L_{i t-1}+\phi^{*} D_{i t-1} * \Delta L L_{i t-1}-\alpha_{2} * D_{i t-1} * \Delta L L_{i t-1}+\varepsilon_{i t} \\
\text { Juntando os termos que contêm } \alpha_{2}: \\
\Delta L L_{i t}=\alpha_{0}+\alpha_{1} D_{i t-1}+\alpha_{2}\left[\left(1-D_{i t-1}\right)^{*} \Delta L L_{i t-1}\right]+\phi\left(D_{i t-1} * \Delta L L_{i t-1}\right)+\varepsilon_{i t}
\end{gathered}
$$

Com base nessa regressão é possivel testar as hipóteses nula e alternativa, tendo por referência o parâmetro , que é equivalente ao somatório dos coeficientes $a_{2}$ e $a_{3}$.

\subsection{AMOSTRA E COLETA DE DADOS}

Para a realização dos testes empíricos foram utilizados dados das demonstrações financeiras anuais dos bancos listados na BM\&FBovespa e daqueles que têm comitê de auditoria e são, pela Resolução CMN n 3.786/2009, obrigados a divulgar sua posição financeira de acordo com o padrão internacional (IFRS) e local (Cosif), considerando o período entre 2010 e 2014.

As demonstrações financeiras foram obtidas diretamente das páginas eletrônicas de cada um dos bancos, na internet.

Como resultado da pesquisa, a amostra final foi composta por 32 bancos, observando a seguinte distribuição:

a) 20 bancos listados na BM\&FBovespa: ABC Brasil, Alfa, Panamericano, Banese, Banestes, Banrisul, Banco do Brasil, BIC Banco, Banco do Nordeste, Bradesco, BRB, BTG Pactual, Daycoval, Banco Indusval, Banco Itaú, Banco Mercantil, Paraná Banco, Banco Pine, Santander e Sofisa.

b) 21 bancos não listados, mas que são obrigados a constituir Comitê de Auditoria: Caixa Econômica Federal, BNDES, Citibank, HSBC, Banco JPMorgan, Banco Safra, Banco Votorantim, BMG, Banco Volkswagen, Banco Deutsche, BNP Paribas, Banco de Desenvolvimento de Minas Gerais, Banco Fator, Banco Ourinvest, Banco Fibra, Banco GMAC, Banco Morgan Stanley, Banco Societe Generale, Bank of 
America, Credit Suisse e Bancoob compõem o grupo dos bancos que fizeram parte da amostra e não estão listados na BM\&FBovespa.

Só foram consideradas na amostra final as demonstrações banco/período em que houve a divulgação simultânea baseada nos dois modelos contábeis. Assim, por exemplo, se não foi localizada a demonstração em IFRS de um banco em determinado período, a demonstração com base no Cosif foi excluída do estudo. Esse procedimento teve o propósito de assegurar a efetiva comparabilidade entre as observações. Por essa razão, inclusive, o Banco da Amazônia, o Banpará e o Banco da Patagônia, apesar de listados na BM\&FBovespa, foram excluídos do estudo, por não apresentaram concomitantemente as demonstrações no padrão Cosif e IFRS. Adicionalmente, também foram desconsideradas oito observações caracterizadas como outliers - em que a variação do lucro líquido de um período para outro representava mais de $5 \%$ dos ativos totais da instituição.

\section{RESULTADOS}

Considerando que a pesquisa busca identificar qual dos modelos contábeis adotados pelas instituições bancárias brasileiras para divulgar suas demonstrações financeiras - Cosif ou IFRS - apresenta de forma mais relevante a característica do conservadorismo contábil condicional, foram constituídas as variáveis necessárias à estimação do modelo (3.1), considerando os parâmetros de definição da amostra destacados na Seção 3.2.

Deve-se ressaltar que o número de observações consideradas na estimação dos modelos acabou sendo restrito, devido aos seguintes motivos: (i) os bancos só passaram a divulgar demonstrações em IFRS a partir de 2010; (ii) nem todos os bancos são obrigados a divulgar esse tipo de demonstração; (iii) no primeiro ano de divulgação em IFRS, nem todos os bancos divulgaram informações comparativas relativas a 2009; e (iv) o modelo utiliza variáveis representativas de variações do lucro líquido e medidas defasadas. Com isso, apesar de considerar informações de 41 bancos, os testes de estimação do modelo de referência contaram com apenas 117 observações, o que representa, em si, uma limitação do estudo. Para compensar essa limitação, foram adotados procedimentos que procuram assegurar a robustez das evidências empíricas apuradas.

Os testes empíricos compreendem a estimação do modelo para identificação do conservadorismo condicional nas demonstrações elaboradas com base nos padrões Cosif e IFRS, com o uso de dados em painel, cujos resultados são apresentados separadamente nas sessões 4.1 e 4.2, respectivamente.

A estimação das regressões com os dados das demonstrações Cosif e IFRS foi realizada com efeitos fixos seccionais, que controlam a heterogeneidade individual entre as instituições, e duplos efeitos fixos, que consideram a heterogeneidade tanto das instituições quanto dos períodos analisados. Adicionalmente, a estimação das regressões foi realizada por mínimos quadrados ordinários (OLS), por matriz de covariância de White e por Cross-Section SUR (PCSE) - os dois últimos com a finalidade de gerar erros-padrões robustos e de permitir heteroscedasticidade e erros contemporaneamente correlacionados, respectivamente. Por fim, o tamanho da amostra permite o relaxamento da premissa de normalidade dos resíduos, de acordo com o Teorema do Limite Central.

\subsection{ESTIMAÇÃO DO CONSERVADORISMO CONDICIONAL NAS DEMONSTRAÇÕES NO PADRÃO IFRS}

Na Tabela 1 são apresentados os resultados da estimação do modelo (3.1) para testar a hipótese do conservadorismo condicional em bancos nas demonstrações elaboradas de acordo com o padrão IFRS. 
Tabela 1: Resultados de regressão com efeitos fixos seccionais e duplos efeitos fixos para identificação do conservadorismo condicional em bancos, nas demonstraçôes elaboradas de açordo com o padräo IFRS

\begin{tabular}{|c|c|c|c|c|c|c|}
\hline \multicolumn{7}{|c|}{$\begin{array}{c}\text { Modelo testado: } \\
\Delta L L_{i t}=\alpha_{0}+\alpha_{1} D_{i t-1}+\alpha_{2} \Delta L L_{i t-1}+\alpha_{3}\left(D_{i t-1} * \Delta L L_{i t-1}\right)+\varepsilon_{i t}\end{array}$} \\
\hline & \multicolumn{3}{|c|}{ Efeitos Fixos Seccionais } & \multicolumn{3}{|c|}{ Duplo Efeitos Fixos } \\
\hline & OLS & White & $\operatorname{SUR}(\mathrm{PCSE})$ & OLS & White & SUR(PCSE) \\
\hline$a_{0}$ & $\begin{array}{r}-0,0042 \\
(0,004) \\
* *\end{array}$ & $\begin{array}{r}-0,042 \\
(0,000) \\
* * * *\end{array}$ & $\begin{array}{r}-0,0042 \\
(0,001) \\
* * * *\end{array}$ & $\begin{array}{r}-0,0041 \\
(0,006) \\
* * *\end{array}$ & $\begin{array}{r}-0,0041 \\
(0,000) \\
* * *\end{array}$ & $\begin{array}{r}-0,0041 \\
(0,031) \\
* *\end{array}$ \\
\hline$a_{1}$ & $\begin{array}{r}-0,0013 \\
(0,523) \\
\end{array}$ & $\begin{array}{r}-0,0013 \\
(0,4925)\end{array}$ & $\begin{array}{r}-0,0013 \\
(0,391)\end{array}$ & $\begin{array}{r}-0,0014 \\
(0,518)\end{array}$ & $\begin{array}{r}-0,0014 \\
(0,500)\end{array}$ & $\begin{array}{r}-0,0014 \\
(0,417)\end{array}$ \\
\hline$a_{2}$ & $\begin{array}{l}0,3959 \\
(0,091)\end{array}$ & $\begin{array}{l}0,3959 \\
(0,056)\end{array}$ & $\begin{aligned} 0,3959 \\
(0,029) \\
* *\end{aligned}$ & $\begin{array}{l}0,3925 \\
(0,102)\end{array}$ & $\begin{array}{r}0,3925 \\
(0,066) \\
*\end{array}$ & $\begin{array}{l}0,3925 \\
(0,034) \\
* *\end{array}$ \\
\hline$a_{3}$ & $\begin{array}{r}-1,3013 \\
(0,000) \\
* * *\end{array}$ & $\begin{array}{r}-1,3013 \\
(0,000) \\
* * *\end{array}$ & $\begin{array}{r}-1,3013 \\
(0,000) \\
* * *\end{array}$ & $\begin{array}{r}-1,2952 \\
(0,000) \\
* * *\end{array}$ & $\begin{array}{r}-1,2952 \\
(0,000) \\
* * *\end{array}$ & $\begin{array}{r}-1,2952 \\
(0,000) \\
* * *\end{array}$ \\
\hline$a_{2}+a_{3}$ & $-0,9054$ & $-0,9054$ & $-0,9054$ & $-0,9027$ & $-0,9027$ & $-0,9027$ \\
\hline № bancos: & 39 & 39 & 39 & 39 & 39 & 39 \\
\hline № observaçōes: & 117 & 117 & 117 & 117 & 117 & 117 \\
\hline $\mathrm{R}^{2}$ & 0,4772 & 0,4772 & 0,4772 & 0,4784 & 0,4784 & 0,4784 \\
\hline$R^{2}$ ajustado & 0,1914 & 0,1914 & 0,1914 & 0,1597 & 0,1597 & 0,1597 \\
\hline F Estatística & 1,6697 & 1,6697 & 1,6697 & 1,5011 & 1,5011 & 1,5011 \\
\hline$F(p$-valor) & 0,0272 & 0,0272 & 0,0272 & 0,0622 & 0,0622 & 0,0622 \\
\hline
\end{tabular}

Onde: $\triangle L L$ corresponde à variação do lucro líquido anual; $D$ é a variável dummy, que assume valor 1 quando $\triangle L L$ e negativa e 0 para os demais; e $\varepsilon$ é o termo de erro com a premissa de distribuição normal e variância constante. Nível de significância: *** 1\%; ** 5\%; * 10\%. P-valores entre parênteses.

Os resultados das regressões baseadas nas demonstrações financeiras elaboradas no padrão IFRS mostram um coeficiente estimado a 2 positivo nas seis combinações testadas - efeitos fixos seccionais e duplos efeitos fixos, combinados com estimação em OLS, matriz de covariância de White e SUR (PCSE) - e estatisticamente relevante a $10 \%$ de significância em cinco dessas combinações, o que indica que os resultados positivos são persistentes. Isso atende à primeira condição do conservadorismo contábil condicional, que pressupõe que os resultados positivos são constantes.

Além disso, o coeficiente a3 apresentou sinal negativo e relevante em todos os testes, implicando em evidências suficientes para afirmar que as variações negativas são transitórias, atendendo à segunda premissa do conservadorismo condicional.

Em relação à terceira condição testada, $a_{2}+a_{3}<0$, o somatório demonstrado na Tabela 1 apresenta sinal negativo em todas as seis estimações realizadas, o que é uma primeira evidência de confirmação da característica do conservadorismo condicional. A confirmação efetiva depende, porém, da verificação da relevância estatística dos valores apurados. Para isso, é estimado o modelo (3.3), onde o somatório dos coeficientes de interesse $\left(a_{2}+a_{3}\right)$ é substituído pelo parâmetro $\phi$. A Tabela 2 apresenta os resultados dessas estimações.

Tabela 2: Estimação do somatório de a2 e a3 (parâmetro ) da Tabela 2, para testar a hipótese nula de que seja igual a zero, considerando os métodos de efeitos fixos seccionais e duplo efeitos fixos

\begin{tabular}{|l|l|l|l|l|l|l|l|}
\hline \multicolumn{7}{|c|}{ Modelo testado: } \\
\hline \multicolumn{7}{|c|}{$\Delta L L_{i t}=\alpha_{0}+\alpha_{1} D_{i t-1}+\alpha_{2}\left(\left(1-D_{i t-1}\right) * L L_{i t-1}\right)+\phi\left(D_{i t-1} * \Delta L L_{i t-1}\right)+\varepsilon_{i t}$} \\
\hline & \multicolumn{1}{|l|}{ Efeitos Fixos Seccionais } & \multicolumn{1}{|l|}{ Duplo Efeitos Fixos } \\
\hline Parâmetro
\end{tabular}


Como as estatísticas t apuradas nas seis estimações (entre -4,9348 a -2,6328) foram menores que o t crítico unicaudal a $5 \%$ de significância, a hipótese nula de que o somatório dos coeficientes $a_{2}$ e $a_{3}$ é igual a zero é rejeitada, ou seja, há evidências de que $a_{2}+a_{3}<0$.

A combinação desse conjunto de resultados permite concluir que as demonstrações financeiras das instituições bancárias elaboradas de acordo com o padrão IFRS atendem às três condições necessárias para a identificação da característica do conservadorismo contábil condicional: coeficiente $a_{2}$ positivo, com valores estatisticamente relevantes ou não diferentes de zero; coeficiente $a_{3}$ negativo; e somatório dos coeficientes $a_{2}$ e $a_{3}$ menor que zero.

\subsection{ESTIMAÇÃO DO CONSERVADORISMO CONDICIONAL NAS DEMONSTRAÇÕES NO PADRÃO COSIF}

Os resultados da aplicação do modelo (3.1) com o uso de dados em painel com efeitos fixos seccionais e duplos efeitos fixos para identificação do conservadorismo condicional em bancos, nas demonstrações elaboradas de acordo com o padrão Cosif estão dispostos na Tabela 3.

Tabela 3: Resultados de regressão com efeitos fixos seccionais e duplos efeitos fixos para identificação do conservadorismo condicional em bancos, nas demonstrações elaboradas de acordo com o padrão Cosif

\begin{tabular}{|c|c|c|c|c|c|c|}
\hline \multicolumn{7}{|c|}{$\begin{array}{c}\text { Modelo testado: } \\
\Delta L L_{i t}=\alpha_{0}+\alpha_{1} D_{i t-1}+\alpha_{2} \Delta L L_{i t-1}+\alpha_{3}\left(D_{i t-1} * \Delta L L_{i t-1}\right)+\varepsilon_{i t}\end{array}$} \\
\hline & \multicolumn{3}{|c|}{ Efeitos Fixos Seccionais } & \multicolumn{3}{|c|}{ Duplo Efeitos Fixos } \\
\hline & OLS & White & SUR(PCSE) & OLS & White & SUR(PCSE) \\
\hline$a_{0}$ & $\begin{array}{r}-0,0025 \\
(0,103)\end{array}$ & $\begin{array}{r}-0,0025 \\
(0,197)\end{array}$ & $\begin{array}{r}-0,0025 \\
(0,202)\end{array}$ & $\begin{array}{r}-0,0020 \\
(0,194)\end{array}$ & $\begin{array}{r}-0,0020 \\
(0,258)\end{array}$ & $\begin{array}{r}-0,0020 \\
(0,348)\end{array}$ \\
\hline$a_{1}$ & $\begin{array}{r}-0,0036 \\
(0,098) \\
*\end{array}$ & $\begin{array}{r}-0,0036 \\
(0,151)\end{array}$ & $\begin{array}{r}-0,0036 \\
(0,126)\end{array}$ & $\begin{array}{r}-0,0044 \\
(0,060) \\
*\end{array}$ & $\begin{array}{r}-0,0044 \\
(0,170)\end{array}$ & $\begin{array}{r}-0,0044 \\
(0,128)\end{array}$ \\
\hline$a_{2}$ & $\begin{array}{r}-0,1608 \\
(0,417)\end{array}$ & $\begin{array}{r}-0,1608 \\
(0,019) \\
* *\end{array}$ & $\begin{array}{r}-0,1608 \\
(0,130)\end{array}$ & $\begin{array}{r}-0,1757 \\
(0,382)\end{array}$ & $\begin{array}{r}-0,1757 \\
(0,010) \\
* *\end{array}$ & $\begin{array}{r}-0,1757 \\
(0,123)\end{array}$ \\
\hline$a_{3}$ & $\begin{array}{r}-0,7802 \\
(0,006) \\
* * *\end{array}$ & $\begin{array}{r}-0,7802 \\
(0,000) \\
* * *\end{array}$ & $\begin{array}{r}-0,7802 \\
(0,072) \\
*\end{array}$ & $\begin{array}{r}-0,7625 \\
(0,008) \\
* * *\end{array}$ & $\begin{array}{r}-0,7625 \\
(0,000) \\
* * *\end{array}$ & $\begin{array}{r}-0,7625 \\
(0,095) \\
*\end{array}$ \\
\hline$a_{2}+a_{3}$ & $-0,9410$ & $-0,9410$ & $-0,9410$ & $-0,9382$ & $-0,9382$ & $-0,9382$ \\
\hline № bancos: & 39 & 39 & 39 & 39 & 39 & 39 \\
\hline № observações: & 117 & 117 & 117 & 117 & 117 & 117 \\
\hline $\mathrm{R}^{2}$ & 0,5605 & 0,5605 & 0,5605 & 0,5770 & 0,5770 & 0,5770 \\
\hline $\mathrm{R}^{2}$ ajustado & 0,3202 & 0,3202 & 0,3202 & 0,3185 & 0,3185 & 0,3185 \\
\hline F Estatística & 2,3329 & 2,3329 & 2,3329 & 2,2322 & 2,2322 & 2,2322 \\
\hline$F$ (p-valor) & 0,0007 & 0,0007 & 0,0007 & 0,0012 & 0,0012 & 0,0012 \\
\hline
\end{tabular}

Os testes efetuados com as demonstrações financeiras no Padrão Cosif, nas seis combinações - efeitos fixos seccionais e duplos efeitos fixos, combinados com estimação em OLS, matriz de covariância de White e SUR (PCSE) - apresentaram coeficiente $a_{2}$ negativo, embora não significantes em quatro dos seis testes. Parâmetros negativos, embora não significantes na maioria dos testes, não atende a uma das premissas do conservadorismo contábil condicional, indicando que o reconhecimento dos ganhos econômicos não se manteve ao longo do tempo, ou seja, não se mostraram persistentes. 
No caso do coeficiente $a_{3^{\prime}}$ foram identificados valores negativos nas seis combinações de testes, com parâmetros estatisticamente relevantes em todos eles. Esses resultados apresentados para o coeficiente a 3 denotam, de forma geral, a existência de transitoriedade nas variações negativas do resultado contábil, sugerindo que perdas econômicas (as más notícias) são reconhecidas tempestivamente e são transitórias, confirmando uma das premissas do conservadorismo condicional.

Considerando que a primeira condição para a confirmação do conservadorismo condicional - parâmetro $a_{2}$ positivo relevante ou não diferente de zero - não foi atendida, resta prejudicada a necessidade de verificação da condição de que a soma de ambos coeficientes seja inferior a zero $\left(a_{2}+a_{3}\right.$ $<0$ ). Ou seja, a condição do conservadorismo condicional nas demonstrações no padrão Cosif não é confirmada. Ademais, tendo em vista que o parâmetro $a_{2}$ apresentou sinal negativo e que a condição de $a_{3}$ ser negativo é uma das premissas do conservadorismo condicional, parece óbvio que, mesmo se constatando que a soma dos parâmetros $a_{2}$ e $a_{3}$ seja menor que zero, isso poderia ser interpretado como uma evidência espúria em relação à condição do conservadorismo.

\subsection{ANÁLISE EM RELAÇÃO ÀS HIPÓTESES DE PESQUISA}

Com base no conjunto dos testes realizados, comentados nas seções 4.1 e 4.2, a primeira premissa que sustenta a hipótese $\mathrm{H}_{1}$ - a de que as demonstrações financeiras anuais das instituições bancárias brasileiras apresentadas em IFRS apresentam as características do conservadorismo condicional - foi confirmada. Em síntese, os resultados empíricos demonstram que nas demonstrações em IFRS os ganhos econômicos são persistentes e as perdas econômicas transitórias, tendo seu reconhecimento feito de forma tempestiva.

Por outro lado, não foram constatadas características de conservadorismo contábil condicional nas demonstrações financeiras elaboradas de acordo com o padrão Cosif, conforme demonstrado na seção 4.2, tendo em vista a não persistência dos ganhos econômicos, embora a transitoriedade das perdas econômicas também tenha sido verificada. Essas evidências comprometem a essência da premissa principal que dá sustentação à hipótese $\mathrm{H}_{2^{\prime}}$ ou seja, a presença do conservadorismo condicional nas demonstrações elaboradas com o padrão Cosif.

A combinação das evidências de conservadorismo condicional nas demonstrações em IFRS e da não identificação dessas mesmas características nas demonstrações produzidas com base no Cosif levam, automaticamente, à não rejeição da hipótese de pesquisa $\mathrm{H}_{1}$, que previa que as demonstrações financeiras anuais das instituições bancárias brasileiras apresentadas em padrão IFRS são mais conservadoras que as demonstrações elaboradas no padrão Cosif, e à automática rejeição da hipótese $\mathrm{H}_{2}$.

Infere-se dos resultados apurados que as demonstrações elaboradas com base no padrão IFRS atingem seu objetivo principal de fornecer informações não viesadas aos usuários, atendendo a um dos requisitos de qualidade da informação contábil, que é a característica do conservadorismo. Por outro lado, não foi confirmada a presença do conservadorismo condicional nas demonstrações elaboradas de acordo com o padrão Cosif, contrariando evidências anteriores encontradas em Dantas, Paulo e Medeiros (2013), por exemplo. Esses resultados contraditórios podem ser explicados principalmente pela abrangência da amostra. Enquanto Dantas, Paulo e Medeiros (2013) utilizaram dados trimestrais de 224 instituições financeiras, no período de 2001 a 2010, o presente se concentra em informações 
anuais de 41 bancos, de 2010 a 2014 - essa restrição é justificada pela escolha metodológica de utilizar apenas as informações para as quais tivesse disponível suas correspondentes no padrão IFRS.

O conjunto dos resultados obtidos no presente estudo é convergente, porém, com os encontrados por Filipin et al. (2012), que analisou o nível de conservadorismo condicional das empresas brasileiras listadas após a adoção das IFRS. Naquele estudo, os autores constataram que as demonstrações financeiras elaboradas segundo o padrão IFRS são mais conservadoras do que as elaboradas segundo as normas brasileiras. A razão para essa confirmação pode estar diretamente relacionada com a essência das normas internacionais, que visam a proteção dos usuários da informação na análise das demonstrações financeiras para subsidiar decisões de investimentos. Filipin et al. (2012) utilizaram como amostra as empresas listadas na BM\&FBovespa no período de 2006 a 2010, excetuando as empresas financeiras, enquanto o presente estudo tem por foco exatamente as instituições financeiras no período entre 2010 e 2014. Em síntese, as amostras dos dois estudos, mesmo sendo completamente diferentes, apresentaram resultados equivalentes.

De qualquer forma, há que se ressaltar as diferenças metodológicas entre dois estudos, pois este compara os dois modelos contábeis em um mesmo período temporal, enquanto Filipin et al. (2012) avaliaram a mudança dos padrões contábeis ao longo do tempo.

\section{CONSIDERAÇÕES FINAIS}

Apesar do tema conservadorismo contábil ser apreciado com relativa frequência na literatura, este estudo explora um aspecto diferenciado, abordando a dicotomia da dupla evidenciação contábil na indústria bancária brasileira, com base em modelos contábeis distintos. O objetivo foi identificar qual modelo - Cosif ou IFRS - produz demonstrações em que a característica do conservadorismo condicional é mais relevante.

Os resultados realizados apresentaram evidências de que, na indústria bancária brasileira, as demonstrações em IFRS apresentam, de forma mais relevante, a característica do conservadorismo condicional do que as demonstrações elaboradas segundo os padrões do Cosif.

Em relação a eventuais aspectos normativos que possam justificar esses resultados, pode-se especular sobre os efeitos dos modelos de reconhecimento de perdas nas operações de crédito, que representam os principais accruals das entidades bancárias (Kanagaretnam, Lobo, \& Mathieu, 2003). No Cosif, o modelo de perdas definido pela Resolução n 2.682, de 1999, do Conselho Monetário Nacional (CMN), é fortemente influenciado pelo prazo de atraso nas operações, o que cria a possibilidade de o impacto do reconhecimento de perdas se estender ao longo do tempo - quanto maior o atraso, pior o rating e maior o provisionamento de perdas. No caso das demonstrações em IFRS, o modelo de perdas incorridas requer o impairment na medida em que se identificar a evidência objetiva de perda. Nesse caso, o reconhecimento das perdas com determinada operação ocorre em um único momento.

De se ressaltar que a oportunidade de realização de estudo do gênero, em que são analisados dois padrões de divulgação financeira, contemporaneamente, é bem restrita, só sendo possível em situações específicas como a que ocorre no âmbito do sistema bancário brasileiro, em que uma decisão do regulador determinou a divulgação concomitante de dois conjuntos de demonstrações financeiras, elaboradas segundo padrões contábeis distintos.

A pesquisa teve como limitação principal a quantidade reduzida de observações, pelas razões expostas na Seção 4. A possibilidade de ampliação do período amostral, na medida em que mais de- 
monstrações em IFRS forem sendo divulgadas, permitirá se verificar se os resultados aqui demonstrados se confirmam ou representam um aspecto transitoriedade.

A perspectiva de um maior número de observações ao longo do tempo pode possibilitar, inclusive, a realização de testes com utilização de variáveis de controle, como a consideração de as instituições serem de controle nacional ou estrangeiro ou mesmo de controle público ou privado, entre outros, ou até a consideração de modelos alternativos e realização de análise de sensibilidade dos resultados.

\section{REFERÊNCIAS}

Amaral, J. V., Riccio, E. L., \& Sakata, M. C. G. (2012). Conservadorismo Contábil Ainda é Discutido? Revista Universo Contábil, 8(1), 70-85.

Ball, R., \& Shivakumar, L. (2005). Earnings quality in UK private firms: comparative loss recognition timeliness. Journal of Accounting and Economics, 39(1), 83-128.

Ball, R., Kothari, S. P., \& Robin, A. (2000). The effect of international institutional factors on properties of accounting earnings. Journal of accounting and Economics, 29(1), 1-51.

Banco Central do Brasil. (1987). Plano Contábil das Instituições do Sistema Financeiro Nacional [Cosif]. Recuperado em 8 de abril de 2014, de https://www.bcb.gov.br/?COSIF.

Basu, S. (1997) The conservatism principle and the asymmetric timeliness of earnings. Journal of Accounting and Economics, 24(1), 3-37.

Black, B. S. (2001). Strengthening Brazil's Securities Markets. SSRN Working Papers. Recuperado em 9 de junho de 2014, de http://papers.ssrn.com/sol3/papers.cfm?abstract_id=247673.

Brito, G. A. S., Lopes, A. B., \& Coelho, A. C. D. (2012). Conservadorismo nos lucros contábeis dos bancos no Brasil: A influência do controle da estatal. Revista Universo Contábil, 8(4), 19-39.

Brito, G. A. S., \& Martins, E. (2013). Conservadorismo contábil e o custo do crédito bancário no Brasil. Brazilian Business Review, 10(1), 27-48.

Capelletto, L. R. (2006). Mensuração do risco sistêmico no setor bancário com utilização de variáveis contábeis e econômicas. Tese de doutorado, Programa de Pós-Graduação em Contabilidade, Universidade de São Paulo (USP), São Paulo.

Coelho, A. C. D., \& Lima, I. S. (2008). Conservadorismo nos Resultados Contábeis Publicados no Brasil: comparação entre companhias de capital fechado e companhias de capital aberto. Revista Universo Contábil, 4(2), 22-41.

Conselho Federal de Contabilidade (2010). Resolução n 1.282, de 28 de maio de 2010. Dispõe sobre os Princípios Fundamentais de Contabilidade. Recuperado em 23 de agosto de 2014, de www.cfc.org. br/sisweb/sre/docs/RES_1282.doc. 
Conselho Monetário Nacional (2009). Resolução no 3.786, de 24 de setembro de 2009. Dispõe sobre a elaboração e a divulgação de demonstrações contábeis consolidadas com base no padrão contábil internacional emitido pelo International Accounting Standards Board (IASB). Recuperado em 2 de abril de 2014, de http://www.bcb.gov.br/pre/normativos/res/2009/pdf/res_3786_v1_O.pdf.

Dantas, J. A., Paulo, E., \& Medeiros, O. M. (2013). Conservadorismo Condicional na Indústria Bancária Brasileira em Situações de Maior Percepção de Risco. Revista Universo Contábil, 9(2), 83-103.

Dantas, J. A., Rodrigues, F. F., Niyama, J. K., \& Mendes, P. C. M. (2010). Normatização contábil baseada em princípios ou em regras? Benefícios, custos, oportunidades e riscos. Revista Contabilidade e Organizações, 4(9), 3-29.

Filipin, R., Teixeira, S. A., Bezerra, F. A., \& Cunha, P. R. (2012). Análise do nível de conservadorismo condicional das empresas brasileiras listadas na BM\&FBOVESPA após a adoção das IFRS. Revista de Contabilidade e Controladoria, 4(2), 24-36.

Kanagaretnam, K., Lim, C. Y., \& Lobo, G. J. (2014) Influence of National Culture on Accounting Conservatism and Risk-Taking in the Banking Industry. The Accounting Review, 89(3), 1115-1149.

Kanagaretnam, K., Lobo, G. J., \& Mathieu R. (2003). Managerial incentives for income smoothing through bank loan loss provision. Review of Quantitative Finance and Accounting, 20(1), 63-80.

Lei no 11.638, de 28 de dezembro de 2007 (2007). Altera e revoga dispositivos da Lei no 6.404, de 15 de dezembro de 1976, e da Lei no 6.385, de 7 de dezembro de 1976, e estende às sociedades de grande porte disposições relativas à elaboração e divulgação de demonstrações financeiras. Recuperado em 2 de abril de 2014, de http://www.planalto.gov.br/ccivil_03/_ato2007-2010/2007/lei/111638.htm.

Lim, C. Y., Lee, E., Kausar, A., \& Walker, M. (2014). Bank accounting conservatism and bank loan pricing. Journal of Accounting and Public Policy, 33(3), 260-278.

Lopes, A. B. (2002). A Informação contábil e o mercado de capitais. São Paulo, Pioneira Thompson Learning.

Most. K. S. (1982). Accounting Theory. Columbus, USA, Grid Publishing INC.

Nichols, C.D., Wahlen, J. M., \& Wieland, M. M. (2009). Publicly traded versus privately held: implications for conditional conservatism in bank accounting. Review of Accounting Studies, 14(1), 88-122.

Paulo, E., Carvalho, L. N. G., \& Girão, L. F. A. P. (2014). Algumas Questões Sobre a Normatização Contábil Baseada em Princípios, Regras e Objetivos. Revista Evidenciação Contábil \& Finanças, 2(2), 24-39.

Rodrigues, J. M. (2012). Convergência Contábil Internacional: Uma análise da qualidade da informação contábil em razão da adoção dos padrões internacionais de contabilidade editados pelo IASB. Tese de doutorado, Programa Multiinstitucional e Inter-Regional de Pós-graduação em Ciências Contábeis UnB/UFPB/UFRN. Brasilia. 
Santos, L. P. G., Lima, G. A. S. F., Freitas, S. C., \& Lima, I. S. (2011). Efeito da Lei 11.638/07 sobre o Conservadorismo Condicional das empresas listadas BM\&FBOVESPA. Revista Contabilidade e Finanças, 22(56), 174-188.

Sterzeck, G. (2011). Efeito da convergência contábil sobre o conservadorismo das instituições financeiras. Dissertação de Mestrado, Programa de Pós-Graduação em Contabilidade, Universidade de São Paulo (USP), São Paulo.

Tapia, B. A., Sánchez, C. B., Alemán, J. P., \& Fernandez, M. T. T. (2011). Conservadurismo del resultado y riesgo de litigio en el sector bancario. Revista Española de Financiación y Contabilidad, 40(152), 556-585.

Uriel, E. (2013). Introduction to Econometrics. Eletronic text book. Version:09/2013. University of Valencia. Recuperado em 10 de janeiro de 2015, de http://www.uv.es/uriel/libroin.htm.

Watts, R. L. (2003a) Conservatism in accounting part I: explanations and implications. Accounting Horizons, 17(3), 207-221.

Watts, R. L. (2003b) Conservatism in accounting part Il: explanations and implications. Accounting Horizons, 17(4), 287-301.

Santos, L. P. G., Lima, G. A. S. F., Freitas, S. C., \& Lima, I. S. (2011). Efeito da Lei 11.638/07 sobre o Conservadorismo Condicional das empresas listadas BM\&FBOVESPA. Revista Contabilidade e Finanças, 22(56), 174-188.

Sterzeck, G. (2011). Efeito da convergência contábil sobre o conservadorismo das instituições financeiras. Dissertação de Mestrado, Programa de Pós-Graduação em Contabilidade, Universidade de São Paulo (USP), São Paulo.

Tapia, B. A., Sánchez, C. B., Alemán, J. P., \& Fernandez, M. T. T. (2011). Conservadurismo del resultado y riesgo de litigio en el sector bancario. Revista Española de Financiación y Contabilidad, 40(152), 556-585.

Uriel, E. (2013). Introduction to Econometrics. Eletronic text book. Version:09/2013. University of Valencia. Recuperado em 10 de janeiro de 2015, de http://www.uv.es/uriel/libroin.htm.

Watts, R. L. (2003a) Conservatism in accounting part I: explanations and implications. Accounting Horizons, 17(3), 207-221.

Watts, R. L. (2003b) Conservatism in accounting part Il: explanations and implications. Accounting Horizons, 17(4), 287-301. 